\title{
Stereo 3D Image Model Visualization from 2D Images
}

\author{
Patel Hetalben Bipinchandra ${ }^{1}$, Pancholi Bhavna K. ${ }^{2}$ \\ ${ }^{1,2}$ Electrical Department, Faculty of Technology and Engineering, \\ Maharaja Sayajirao University of Baroda (MSU), Vadodara, Gujarat, India
}

\begin{abstract}
D is three dimensions. It is nothing but the derived visual information. Over the time, various techniques have been evolved to enhance the visual information. The problems in these techniques are we cannot see the other sides of the object in single image. And we cannot predict the other sides of any uneven object by single image. So my study is based on making a single 'IMAGE' model having multidimensional details which can be seen by rotation of mouse pointer on the object. There is much software for making $3 D$ model using $2 \mathrm{D}$ images but by using manual process. $3 \mathrm{D}$ image visualization is very useful for the study of any uneven object like tumor, temple, and shaded buildings. Hence, a novel approach has been developed for generation of application which makes multi dimensional view of any single object by using single device without manual process.
\end{abstract}

Keywords: 3D image visualization, operating system (Linux), projections, interpolation, triangulation, and Image reconstruction

\section{Introduction}

3D image visualization from different angles is very useful especially in military, medicine, geography, industry, satellite and remote sensing. 3D image visualization gives very useful information about object to visualize and so that we can observe their properties. There are many methods to make 3D visualization from 2D images.

Reconstruction of 3D image by using ,single ${ }^{e e}$ image is mostly used in animation techniques. In which by changing the pixel values in 2D, image we can make 3D visualization on that single image, but having only single sided information.

Reconstruction of 3D image by using „two "eimages in which we have to take two projection images which process is similar to a pair of two human eyes for viewing images. So the images we get are obtained from the projections of two cameras which are located at different positions. So by using idea of human eyes to view any object having two projections, we can perform 3D visualization of any object with two projection images. Still we cannot get the idea of another side of any uneven object. Because we have only two projection images, we can say the less information of 3D image by using "many" images is very useful in industries and medical. A multi dimensional view of image is a collection of images of the same scene captured by more than two cameras. This method is very useful when we want to observe object properties in all directions. The possibility of 3D visualization in multi dimension is still less developed. So, it is type of research which need new tools, procedures and devices which may useful in development of $3 \mathrm{D}$ visualization process. So here we used Linux operating system which is open source OS.

In this paper the concept is $3 \mathrm{D}$ image visualization which formulates and realizes concepts of different shaped objects. And here we try to make an efficient algorithm to perform 3D image visualization. We have used OpenCV 2.4.1, QT Creator 2.0.1 in Linux OS.

\section{Flow Diagram}

The basic flow diagram is as figure 1. There are three types of image: Virtual, Static and Dynamic.

In this work, algorithms are applied on images of linear virtual object.

\section{BASIC FLOW DIAGRAM}

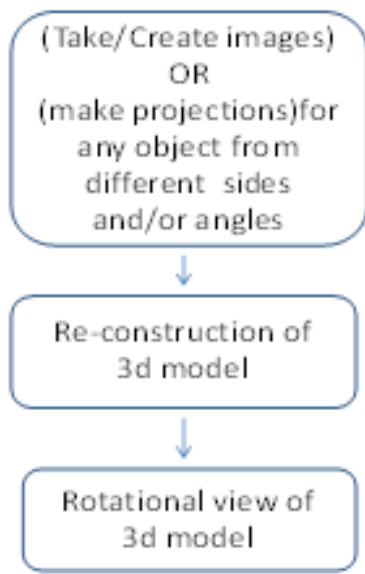

Figure 1

In this work virtual images are taken as a reference images. Then reconstruct 3D object by using interpolation and triangulation methods. Then make multi dimensional view of object in single image by which we can see the properties of object by rotating the mouse point.

If we take static image then we have to first take projections from different angles. Then apply interpolation and triangulation methods to reconstruct object. Then apply same algorithms for multi dimensional visualization.

\section{A. Projections}

In projection technique, projection planes are made by taking projections from different angles, which are shown as camera image in below figure. The feature points are obtained on projection plane from object points. 


\section{International Journal of Science and Research (IJSR) \\ ISSN (Online): 2319-7064}

Index Copernicus Value (2013): 6.14 | Impact Factor (2014): 5.611

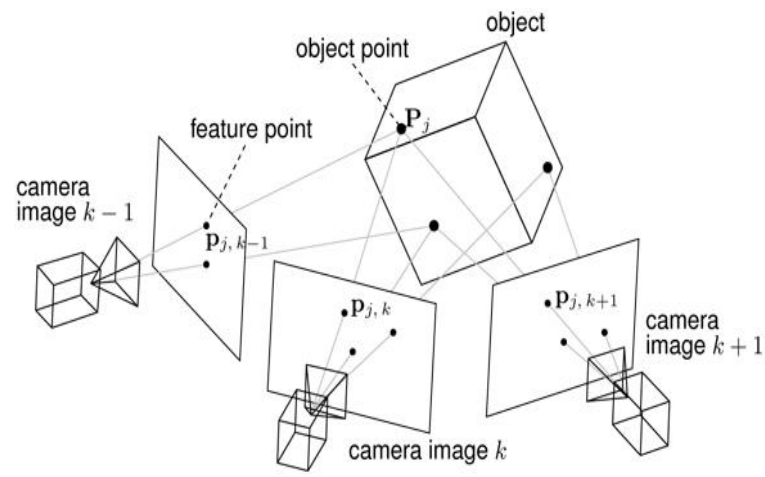

Figure 2

In this work orthographic projection technique is used.

When the views are angle independent then, orthographic projections is useful.

Orthographic projection (or orthogonal projection) is nothing but the representation of a three-dimensional object in two dimensions.

When three dimensional objects are captured by using camera, we always get loss of depth of information about it. There are many situations in which 2D projections are used to reconstruct 3D shape of an object. Engineering objects are represented in three orthographic views: front, top and side views. Most of the CAD tools provide this facility but it is a challenging task to transform manual drawings into CAD representation.

A simple orthographic projection onto the plane $\mathrm{z}=0$ can be defined by the following matrix:

$$
P=\left[\begin{array}{lll}
1 & 0 & 0 \\
0 & 1 & 0 \\
0 & 0 & 0
\end{array}\right]
$$

For each point $\mathrm{v}=(\mathrm{vx}, \mathrm{vy}, \mathrm{vz})$ on the object, the transformed point on projection plane would be

$$
\begin{gathered}
P v=\left[\begin{array}{lll}
1 & 0 & 0 \\
0 & 1 & 0 \\
0 & 0 & 0
\end{array}\right]\left[\begin{array}{l}
v_{x} \\
v_{y} \\
v_{z}
\end{array}\right]=\left[\begin{array}{c}
v_{x} \\
v_{y} \\
0
\end{array}\right] \\
\begin{array}{ccc}
\text { Projection } & \begin{array}{l}
\text { Object } \\
\text { plane }
\end{array} & \begin{array}{l}
\text { Point on } \\
\text { point }
\end{array} \\
\text { Projection } \\
\text { plane }
\end{array}
\end{gathered}
$$

So the projection plane would be like below figure:

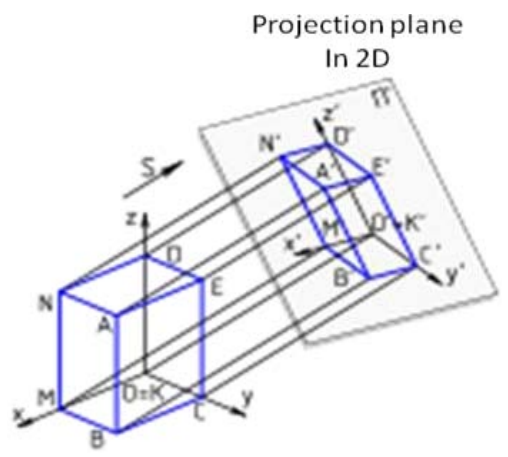

Object 3D
(2)

\section{B. Interpolation}

Interpolation is often used to fill the gaps in reconstruction of an image. There are three types of interpolation technique.

1) Nearest Neighbor

2) Linear

3) Bicubic

Linear interpolation technique is used in this work. In linear interpolation, we take two known points. In figure 4, they are $(\mathrm{x} 0, \mathrm{y} 0)$ and $(\mathrm{x} 1, \mathrm{y} 1)$. The linear interpolant is the straight line between these points.

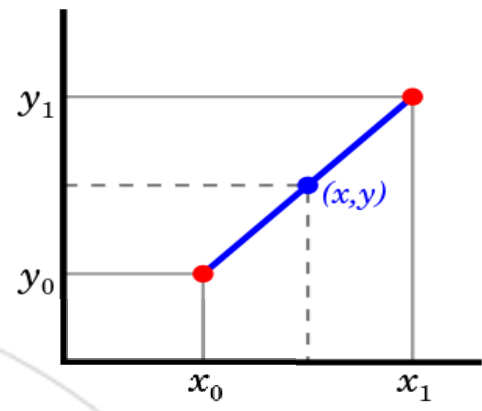

Figure 4

Having the value of $\mathrm{X}$ in the interval $(\mathrm{x} 0, \mathrm{x} 1)$, we can get the value of $\mathrm{Y}$ along the straight line by the below equation.

$$
\frac{y-y_{0}}{x-x_{0}}=\frac{y_{1}-y_{0}}{x_{1}-x_{0}}
$$

We can write above equation for Y:

$$
y=y_{0}+\left(y_{1}-y_{0}\right) \frac{x-x_{0}}{x_{1}-x_{0}}
$$

In below figure, red dots represent linear interpolation and blue line represents pieces of linear interpolants.

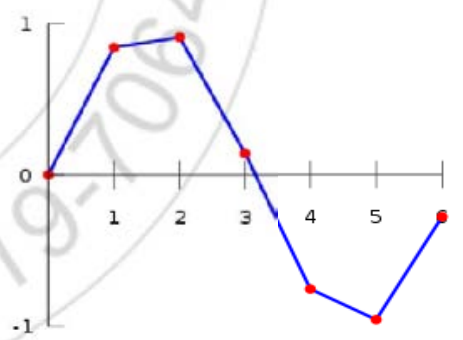

Figure 5

\section{Triangulation}

Triangulation has two functions:

1) Divide the plane

2) Collect or make plane

In this work, triangulation is referred to as reconstruction of 3D image.

If a pair of corresponding points in two, or more images, can be found it must be the case that they are the projection of a common 3D point $x$ i.e. in tetrahedron, if we take projection of 3 planes and remaining plane is not projected, then all planes will be re-constructed and also the remaining plane will be re-constructed as a flat plane by triangulation method.

Figure 3 


\section{International Journal of Science and Research (IJSR) \\ ISSN (Online): 2319-7064}

Index Copernicus Value (2013): 6.14 | Impact Factor (2014): 5.611

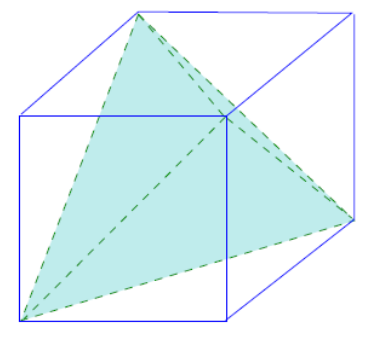

5 Tetrahedra

Figure 6

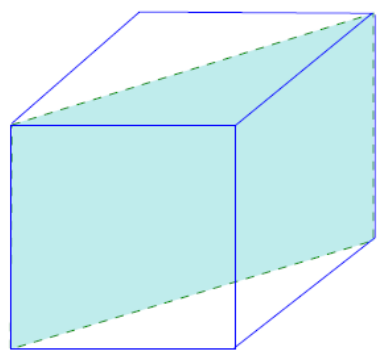

6 Tetrahedra

Figure 7

After applying all these methods and different libraries from Open CV a tetrahedron object is created. This is linear object.

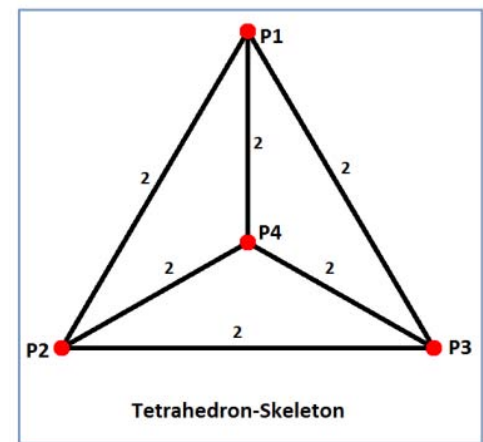

Figure 8

To create four planes A, B, C, D we have took each side having 2 units. We have Four coordinates = p1, p2, p3, p4

We can create each plane by combination of three coordinates.

Plane $A=\{p 1, p 2, p 3\}$

Plane $B=\{p 1, p 3, p 4\}$

Plane $\mathrm{C}=\{\mathrm{p} 1, \mathrm{p} 2, \mathrm{p} 4\}$

Plane $\mathrm{D}=\{\mathrm{p} 2, \mathrm{p} 3, \mathrm{p} 4\}$

If we set this object on 3 co-ordinates so that the middle of any one plane sets on origin $(0,0,0)$, then we get the values of all co ordinates like $\mathrm{p} 1=\{0.0,-1.0,+2.0\}, \mathrm{P} 2=$ $\{+1.73205081,-1.0,-1.0\}, P 3=\{-1.73205081,-1.0,-1.0\}$, $\mathrm{P} 4=\{0.0,+2.0,0.0\}$

Figure 9 shows a plane of virtual object.

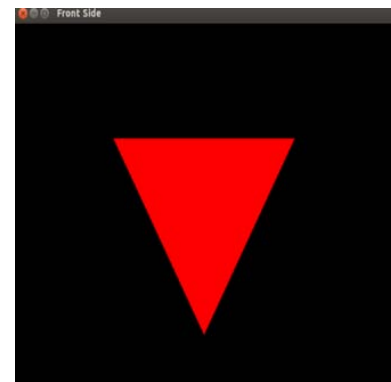

Figure 9

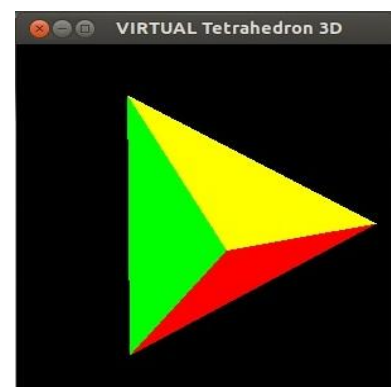

Figure 10

Virtual object tetrahedron is created by combining all planes. Figure 10 shows virtual 3D object. Now, OpenCV libraries are used to rotate the object for stereo view on screen. Figure 11 (a-e) shows stereo view of tetrahedron object at different angles.
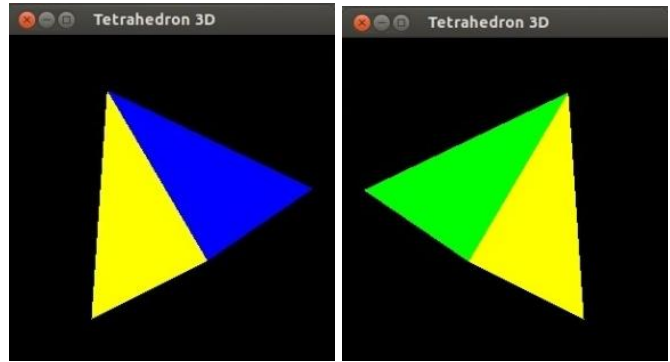

(a) $X=0, Y=0, Z=0$ (b) $X=90, Y=0, Z=0$
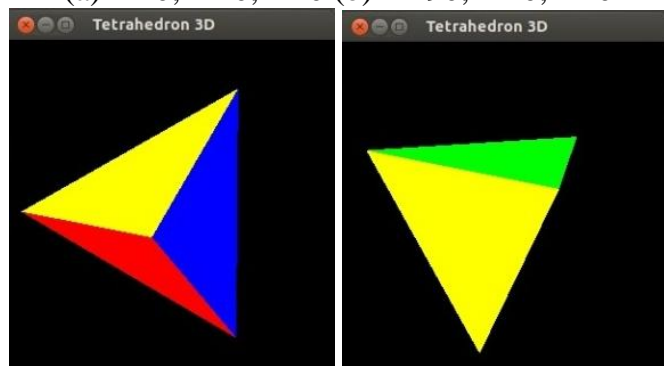

(c) $\mathrm{X}=0, \mathrm{Y}=90, \mathrm{Z}=0$ (d) $\mathrm{X}=0, \mathrm{Y}=0, \mathrm{Z}=90$

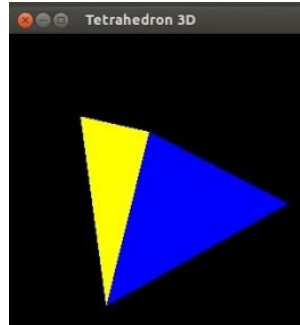

(e) $\mathrm{X}=120, \mathrm{Y}=30, \mathrm{Z}=270$

Figure 11 (a-e) 


\section{Advantage of this Work}

- We can observe multi dimensional view of a single object using single device without manual process.

- It can be implemented on various type of images

- It is very useful to represent uneven objects by using single device.

- Less complex as compare to manual process

\section{Conclusion \& Future Work}

- In this paper, we have described an innovative method of converting a normal 2D image into a 3-dimensional image which can be seen by stereo dimensions. We have successfully made stereo view of a single linear object.

- For future work, stereo view of non linear object and apply algorithms on static image is remaining part of work.

\section{References}

[1] Megha P. Arakeri and G. Ram Mohana Reddy , "An Effective and Efficient Approach to 3D Reconstruction and Quantification of Brain Tumor on Magnetic Resonance Images" International Journal of Signal Processing, Image Processing and Pattern Recognition Vol. 6, No. 3, June, 2013

[2] Priya Pankaj Patel ,Jwolin Patel , M.E, E.C, Rushabh Shah, " $3 \mathrm{~d}$ image generation using interpolation and triangulation methods on arm-9 processor based single board computer platform “. ISSN 2250-0588, Volume 4, Issue 3, March 2014)

[3] J. Poco1,2, R. Etemadpour3, F.V. Paulovich1, T.V. Long3,4, P. Rosenthal3,5, M.C.F. Oliveira1, L. Linsen3 And R., " A Framework For Exploring Multidimensional Data With 3D Projections" Eurographics / IEEE Symposium on Visualization 2011 (EuroVis 2011), Volume 30 (2011), Number 3

[4] Jianhua Yan, Keqi Zhang, Chengcui Zhang, Member, Ieee,shu-ching Chen, Senior Member, IEEE, And Giri Narasimhan, "Automatic Construction Of 3-D Building Model From Airborne LIDAR Data Through 2-D Snake Algorithm" IEEE Transactions On Geoscience And Remote Sensing, Vol. 53, No. 1, January 2015

[5] Min Qi, Jiankui Jia, Yuelei Xu, Ke Li. , "Automatic Extraction of the Building Rectilinear Rooftops Based on Region over Growing", Journal Of Computers, Vol. 9, No. 10, October 2014

[6] Mohammad Izadi, Student Member, IEEE, and Parvaneh Saeedi, Member, IEEE , "Three-Dimensional Polygonal Building Model Estimation From Single Satellite Images",IEEE Transactions On Geoscience And Remote Sensing, Vol. 50, No. 6, June 2012

[7] Opencv.org

[8] QTCreator.org 